\title{
Trend of Invasive Streptococcus agalactiae at Tertiary Care Hospital in Japan
}

\author{
Masaaki Minami ${ }^{*}$, Hideki Nishiyama², Shinobu Ikegami², Takuya Hattori², \\ Norihiro Yuasa', Makoto Kawashima3, Michio Ohta ${ }^{4}$ \\ ${ }^{1}$ Department of Bacteriology, Graduate School of Medical Sciences, Nagoya City University, Nagoya, Japan \\ ${ }^{2}$ Department of Clinical Laboratory, Japanese Red Cross Nagoya Daiichi Hospital, Nagoya, Japan \\ ${ }^{3}$ Department of Clinical Laboratory, Japanese Red Cross Nagoya Daini Hospital, Nagoya, Japan \\ ${ }^{4}$ School of Nursing, Sugiyama Jyogakuen University, Nagoya, Japan \\ Email: "minami@med.nagoya-cu.ac.jp
}

Received 19 January 2016; accepted 19 February 2016; published 26 February 2016

\begin{abstract}
Streptococcus agalactiae causes various severe infectious diseases such as sepsis, meningitis, and streptococcal toxic shock-like syndrome. Though Streptococcus agalactiae infection has been increasing recently, the comprehensive characteristic investigation of invasive Streptococcus agalactiae isolated in tertiary care hospitals has not been nearly performed in Japan. In this study, we investigated the clinical characteristics and antimicrobial susceptible patterns of 88 Streptococcus agalactiae isolated at two tertiary care hospitals during 2009-2015 in Japan. There was no significant differences between genders in our study. Two-third Streptococcus agalactiae were isolated from over age 60 . Total mortality rate was $19 \%$ and invasive Streptococcus agalactiae-associated death cases have occurred every year after 2011. All Streptococcus agalactiae were completely susceptible toampicillin. Total non-susceptible rates of erythromycin, minocycline, levofloxacin and trimethoprim-sulfamethoxazole in this study were approximately $30 \%, 44 \%, 37 \%$, and $7 \%$, respectively. Our results suggest the need for continuous antimicrobial susceptibility survey of Streptococcus agalactiae.
\end{abstract}

\section{Keywords}

Streptococcus agalactiae, Susceptibility, Antimicrobial Resistance, Epidemiology

\section{Introduction}

Streptococcus agalactiae is a gram-positive and encapsulated bacterium, which displays beta-hemolytic activity on blood agar. It is classified in Lancefield group B. As it is part of the commensal flora in the genital and lower gastrointestinal tracts under $40 \%$ of healthy adults [1], Streptococcus agalactiae has been recognized as emerging infection in adults with underlying medical conditions (mainly malignancy and diabetes mellitus) [2]. Previous report demonstrated a significant increase in the disease burden in adults, a trend that might be explained

${ }^{*}$ Corresponding author.

How to cite this paper: Minami, M., Nishiyama, H., Ikegami, S., Hattori, T., Yuasa, N., Kawashima, M. and Ohta, M. (2016) Trend of Invasive Streptococcus agalactiae at Tertiary Care Hospital in Japan. Journal of Biosciences and Medicines, 4, 15-20. http://dx.doi.org/10.4236/jbm.2016.43003 
by a growing number of patients with chronic medical conditions [3]. The mortality rate of Streptococcus agalactiae bacteremia in adults ranges from $9 \%$ to $47 \%$ and is significantly associated with age [4]. Although invasive Streptococcus agalactiae remains exquisitely sensitive to penicillin and other beta lactam antibiotics in Japan [5], the report in Hongkong showed the emergence of penicillin G non-susceptible invasive Streptococcus agalactiae [6]. Furthermore resistance to macrolide, and fluoroquinolone has also been reported over the last 20 years [7]. The loss of efficacy of erythromycin and clindamycin expressed the increasing rates of resistance in Streptococcus agalactiae (32\% and 15\%, respectively) [8]. Although cross-resistance to macrolide and lincosamide antibiotics has been observed among a variety of Streptococcus agalactiae, this multiple-resistance phenomenon has occurred among Streptococcus agalactiae [9]. The present study was conducted to find out the recent clinical also characteristics of invasive Streptococcus agalactiae isolated at two tertiary care hospitals in the central of Japan.

\section{Materials and Methods}

\subsection{Strains and Clinical Data Collection}

A total of 88Streptococcus agalactiae was obtained from aseptically specimens at Japanese Red Cross Nagoya Daiichi Hospital and Japanese Red Cross Nagoya Daini Hospital from 2009 to 2015. Japanese Red Cross Nagoya Daiichi Hospital and Japanese Red Cross Nagoya Daini Hospitalare852-bed and 812-bedtertiary care hospital in the central region of Japan, respectively. We used medical records appended to clinical species for the analysis of clinical feature at Japanese Red Cross Nagoya Daiichi Hospital and Japanese Red Cross Nagoya Daini Hospital. We considered several isolates from the same region of the same patient as one isolate per one patient for the analysis in this study. All streptococcus isolates were identified by standard conventional biochemical methods and conformed by $16 \mathrm{~S}$ ribosomal DNA sequence analysis [10]. Our experimental design was approved by the ethics committee at both hospitals.

\subsection{Antimicrobial Susceptibility Analysis}

Streptococcus agalactiae isolates were examined for typical five antibiotic susceptibilities as ABPC; ampicillin, EM; erythromycin, MINO; minocycline, LVFX; levofloxacin, and ST; trimethoprim-sulfamethoxazole. Minimal inhibitory concentrations (MICs) were determined by E-test. Evaluation of susceptibilities was calculated based on Clinical Laboratory Standard Institute (CLSI) break point [11].

\subsection{Statistical Analysis of the Data}

We conducted the statistical analysis with the chi-squared test or Fisher's exact test when appropriate. Differences were considered significant when $p$ was $<0.05$.

\section{Results}

First of all, we evaluated the relationship between clinical patients' features and Streptococcus agalactiae.

The numbers of Streptococcus agalactiae isolates in both 2011 and 2013 were about four times as same as that in 2009 and 2012. We did not foundany significant differences on the numbers of isolates. The total number of male and female patients was 48 and 40, respectively (Figure 1). Although the number of male patients was larger than that of female patients, there was no significant differences between genders in Streptococcusagalactiae. The age range was categorized as under 18-year age, from 19 to 64 year age, over 65 year age (Figure 2). Totally, the numbers of over 65year's patients (about 66\%) was largest in this study $(p<0.05)$. The number of 10 - 60 years patients was low for 7 years. Although about 10\% of Streptococcus agalactiae isolates were from 0 year's patient, invasive Streptococcus agalactiae were not isolated from 0 year's patient every year in those hospitals. Furthermore, total mortality rate was $19.3 \%$ (17 patients) and 13 cases (76.4\%) were occurred from aged patients among 7 years. After 2011, invasive Streptococcus agalaciate-associated death cases occur every year. Finally, we analyzed the antimicrobial susceptibility of Streptococcus agalactiae in this study. Ampicillin was susceptible against all Streptococcus agalactiae isolates and we did not find any penicillin no susceptible Streptococcus agalactiae. Figure 3 showed the susceptibility of erythromycin against Streptococcus agalactiae. Total non-susceptible rate of erythromycin was about 30\% among 7 years. About one-third of Streptococcus 


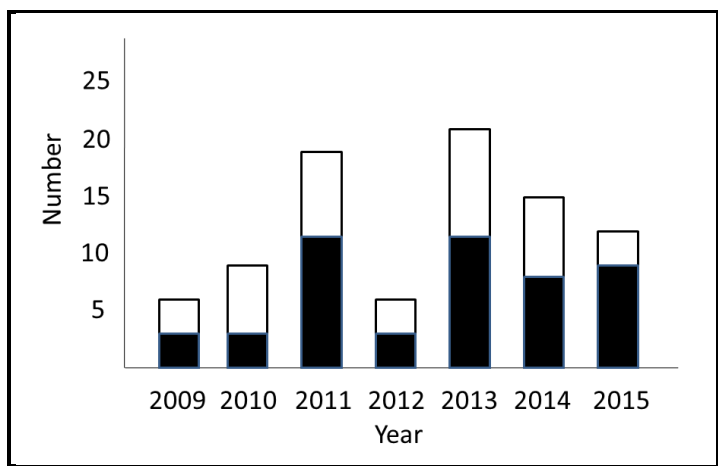

Figure 1. Gender distribution of Streptococcus agalactiae isolates. Black box; male, white box; female.

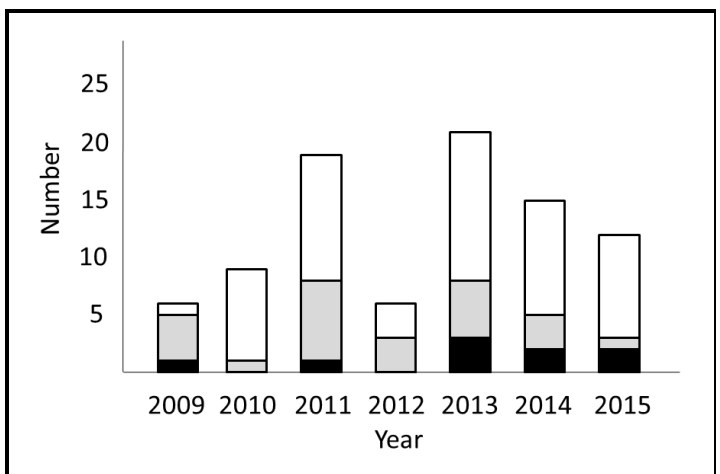

Figure 2. Age distribution of Streptococcus agalactiae isolates. Black box; under 18 year age, gray box; 19 - 64 year age, white box; over 64 year age.

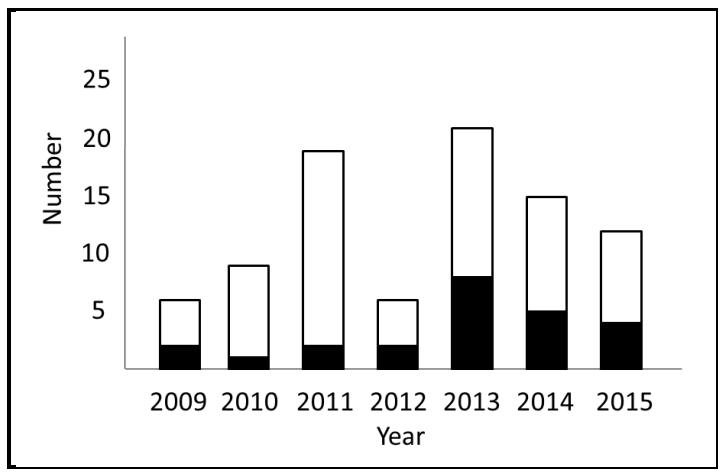

Figure 3. Erythromycin susceptibility of Streptococcus agalactiae isolates. Black box; no susceptible, white box; susceptible.

agalactiae had non-susceptible activity against erythromycin from 2013 to 2015. Figure 4 showed the susceptibility of minocycline against Streptococcus agalactiae. Total non-susceptible rate of erythromycin was about $44 \%$ among 7 years. Over half of Streptococcus agalactiae had non-susceptible activity against erythromycin from 2014 to 2015. Figure 5 showed the susceptibility of levofloxacin against Streptococcus agalactiae. Total nonsusceptible rate of levofloxacin was about 37\% among 7 years. About one-third of Streptococcus agalactiae had no susceptible activity against levofloxacin from 2013 to 2015. Figure 6 showed the susceptibility of trimethoprim-sulfamethoxazole against Streptococcus agalactiae. Total non-susceptible rate oftrimethoprim-sulfamethoxazole was about $7 \%$ among 7 years. At least one Streptococcus agalactiae isolates had no susceptible activity against trimethoprim-sulfamethoxazole from 2013 to 2015. 


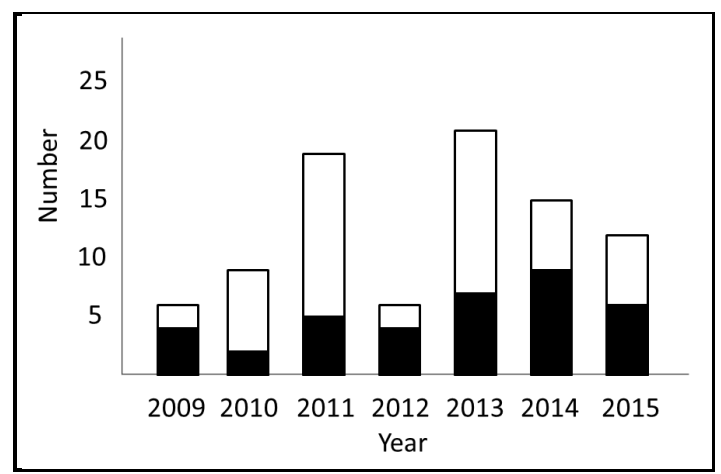

Figure 4. Minocyclinesusceptibility of Streptococcus agalactiae isolates. Black box; no susceptible, white box; susceptible.

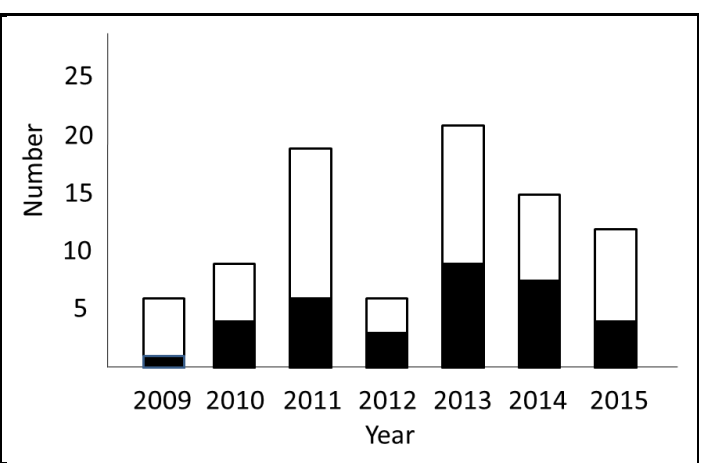

Figure 5. Levofloxacinsusceptibility of Streptococcus agalactiae isolates. Black box; no susceptible, white box; susceptible.

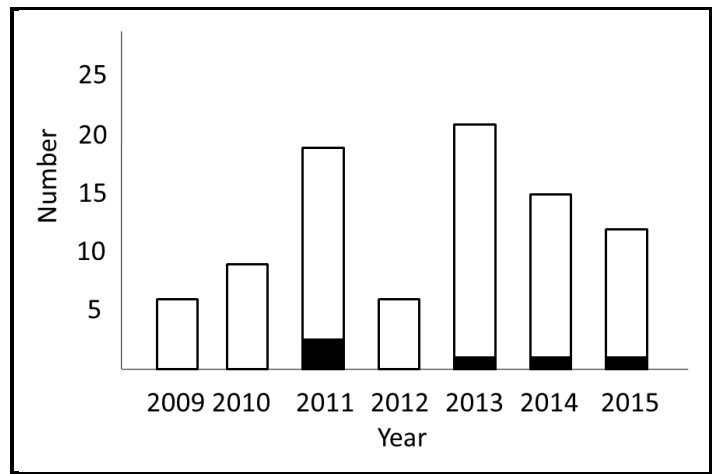

Figure 6. Trimethoprim-sulfamethoxazolesusceptibility of Streptococcus agalactiae isolates. Black box; no susceptible, white box; susceptible.

\section{Discussion}

In this study, we described the clinical characteristics and antimicrobial susceptible patterns of all Streptococcus agalactiae isolated from two tertiary carehospitals in the central of Japan among recent 7 years. Although we have little interest of Streptococcus agalactiae as compared with Streptococcus pyogenes before, this study may imply that Streptococcus agalactiae will increase gradually from now on. With respect to gender group, the number of isolation in female patients was almost same as that in male patients. This result is almost consistent with previous report [3] [5]. Next, we clarified Streptococcus agalactiae with age distribution.

Although 0 years aged-patient caused Streptococcus agalactiae infection, the numbers were relative low and the death cases were nothing among 7 years. The children and young adults did not cause invasive streptococcal 
disease. Patients over 65-years frequently caused Streptococcus agalactiae infection. As we have recognized Streptococcus agalactiae as serious infectious disease for female and neonate, we may reevaluate Streptococcus agalactiae as serious infection disease for aged-people. With respect to mortality, at least one patient caused severe Streptococcus agalactiae infection died ever year. Although we do not definestreptococcal toxic shock-like syndrome cases in this study, the death cases may almost consistent with streptococcal toxic shock-like syndrome according to CDC criteria [13]. Because those death cases were included in (1) aseptic site, (2) septic shock, and (3) multiple organ. This result may also support that streptococcal toxic shock-like syndrome caused by Streptococcus agalactiae gradually increased in Japan. With respect to antimicrobial susceptibility, we did not find penicillin non-susceptible Streptococcus agalactiae in this study. Indeed, penicillin non-susceptible Streptococcus agalactiae has not been isolated from aseptic site in Japan before [5] [12]. Previous report showed that the macrolide non-susceptible rates of Streptococcus agalactiae was about 13\% [14]. Recently macrolide have been used frequently against respiratorytract infectious disease and the emergence of macrolide resistant Streptococcus pneumonia has increased and the more consumption of macrolide induced more macrolide resistance in Japan [15]. This fact may also apply to Streptococcus agalactiae cases. Actually, the total macrolide non-susceptible rate of Streptococcus agalactiaewas about 30\% in our study. Previous report demonstrated that the tetracycline non-susceptiblerates of Streptococcus agalactiae were about 47\% [14]. Our study revealed that minocycline non-susceptible rates of Streptococcus agalactiae were about $44 \%$. We assumed that tetracycline such as minocycline resistant rate has not increased significantly. Levofloxacin non-susceptible rates of Streptococcus agalactiae were about $19 \%$ in former study [14]. As fluoroquinolone has been widely used genitourinary disease, digestive disease and respiratory disease [16]. We assume that the change of fluoroquinolone resistance among Streptococcus agalactiae spread worldwide in future. This suggested that levofloxacin nonsusceptible rate of Streptococcus agalactiae was about $40 \%$ in our study. From these views, we considered the further necessity of the analysis of Streptococcus agalactiae strains. Thus, we need further antimicrobial surveillance to prevent the spread of antimicrobial resistant Streptococcus agalactiae.

\section{Conclusion}

In summary, we clarified the characteristics of invasive Streptococcus agalactiae in two tertiary care hospitals in the central of Japan. Although several antibiotics such as penicillin are effective against Streptococcus agalactiae, incidence of invasive Streptococcus agalactiae infection is not significant decreasing. Severe invasive Streptococcus agalactiae disease such as streptococcus toxic shock-like syndrome may occur by dissemination to other organs of the body if not treated adequately. Our investigation aims is not only to reduce the morbidity and mortality in the patients but also to control the emergence and spread of resistance among Streptococcus agalactiae. The results from our study strongly suggest the need for epidemiological surveillance of antibiotic resistant pathogen.

\section{Acknowledgements}

We thank Mr. Masashi Ishihara and Ms. Miwako Fujimura for special encouragement. This study was supported by a grant-in-aid for research from the Nagoya City University, Japan.

\section{References}

[1] Dhanoa, A., Karunakaran, R. and Puthucheary, S.D. (2010) Serotype Distribution and Antibiotic Susceptibility of Group B Streptococci in Pregnant Women. Epidemiol Infect, 138, 979-981. http://dx.doi.org/10.1017/S0950268809991105

[2] Skoff, T.H., Farley, M.M., Petit, S., Craig, A.S., Schaffner, W., Gershman, K., et al. (2009) Increasing Burden of Invasive Group B Streptococcal Disease in Nonpregnant Adults, 1990-2007. Clin Infect Dis., 49, 85-92. http://dx.doi.org/10.1086/599369

[3] Al Akhrass, F., Abdallah, L., Berger, S., Hanna, R., Reynolds, N., Thompson, S., et al. (2013) Streptococcus agalactiae Toxic Shock-Like Syndrome: Two Case Reports and Review of the Literature. Medicine (Baltimore), 92, 10-14. http://dx.doi.org/10.1097/MD.0b013e31827dea11

[4] Colford Jr., J.M., Mohle-Boetani, J. and Vosti, K.L. (1995) Group B Streptococcal Bacteremia in Adults. Five Years' Experience and a Review of the Literature. Medicine (Baltimore), 74,176-190. http://dx.doi.org/10.1097/00005792-199507000-00002 
[5] Ikebe, T., Chiba, K., Shima, T., Masuda, C., Okuno, R., Ohya, H., et al., Working Group for Beta-Hemolytic Streptococci in Japan (2015) Evaluation of Streptococcal Toxic Shock-Like Syndrome Caused by Group B Streptococcus in Adults in Japan between 2009 and 2013. J Infect Chemother, 21, 207-211. http://dx.doi.org/10.1016/j.jiac.2014.12.002

[6] Chu, Y.W., Tse, C., Tsang, G.K., So, D.K., Fung, J.T. and Lo, J.Y. (2007) Invasive Group B Streptococcus Isolates Showing Reduced Susceptibility to Penicillin in Hong Kong. J Antimicrob Chemother, 60, 1407-1409. http://dx.doi.org/10.1093/jac/dkm390

[7] Wang, Y.H., Chen, C.L., Hou, J.N., Wang, Y.R., Lin, T.Y., Wang, M.H., et al. (2015) Serotype Distribution and Resistance Genes Associated with Macrolide and Fluoroquinolone Resistance in Streptococcus agalactiae Isolates from a Hospital in Southern Taiwan. Biomed Journal, 38, 215-220. http://dx.doi.org/10.4103/2319-4170.138306

[8] Phares, C.R., Lynfield, R., Farley, M.M., Mohle-Boetani, J., Harrison, L.H., Petit, S., et al., Active Bacterial Core Surveillance/Emerging Infections Program Network (2008) Epidemiology of Invasive Group B Streptococcal Disease in the United States, 1999-2005. JAMA, 299, 2056-2065. http://dx.doi.org/10.1001/jama.299.17.2056

[9] Banno, H., Kimura, K., Tanaka, Y., Kitanaka, H., Jin, W., Wachino, J., et al. (2014) Characterization of MultidrugResistant Group B Streptococci with Reduced Penicillin Susceptibility Forming Small Non-Beta-Hemolytic Colonies on Sheep Blood Agar Plates. J Clin Microbiol., 52, 2169-2171. http://dx.doi.org/10.1128/JCM.00226-14

[10] Hashikawa, S., Iinuma, Y., Furushita, M., Ohkura, T., Nada, T., Torii, K., et al. (2004) Characterization of Group C and G Streptococcal Strains That Cause Streptococcal Toxic Shock Syndrome. J Clin Microbiol., 42, 186-192. http://dx.doi.org/10.1128/JCM.42.1.186-192.2004

[11] Clinical and Laboratory Standards Institute (CLSI) (2014) Performance Standards for Antimicrobial Susceptibility Testing: 24st Informational Supplement. Clinical and Laboratory Standards Institute M100-S24, Wayne.

[12] Kimura, K., Nishiyama, Y., Shimizu, S., Wachino, J., Matsui, M., Suzuki, S., et al. (2013) Screening for Group B Streptococci with Reduced Penicillin Susceptibility in Clinical Isolates Obtained between 1977 and 2005. Jpn J Infect Dis., 66, 222-225. http://dx.doi.org/10.7883/yoken.66.222

[13] The Working Group on Severe Streptococcal Infections (1993) Defining the Group A Streptococcal Toxic Shock Syndrome. Rationale and Consensus Definition. JAMA, 269, 390-391. http://dx.doi.org/10.1001/jama.1993.03500030088038

[14] Ueno, H., Yamamoto, Y., Yamamichi, A., Kikuchi, K., Kobori, S. and Miyazaki, M. (2012) Characterization of Group B Streptococcus Isolated from Women in Saitama City, Japan. Jpn J Infect Dis., 65, 516-521. http://dx.doi.org/10.7883/yoken.65.516

[15] Minami, M., Sakakibara, R., Imura, T., Morita, H., Kanemaki, N. and Ohta, M. (2014) Prevalence and Antimicrobial Susceptibility Pattern of Streptococcus Pneumoniae at General Hospital in the Central Region of Japan from December 2013 to February 2014. J. Biosciences. Medicine, 2, 12-17.

[16] Davis, R. and Bryson, H.M. (1994) Levofloxacin. A Review of Its Antibacterial Activity, Pharmacokinetics and Therapeutic Efficacy. Drugs, 47, 677-700. http://dx.doi.org/10.2165/00003495-199447040-00008 